\title{
Properties of the Slab Modes in Photonic Crystal Optical Waveguides
}

\author{
Ali Adibi, Yong Xu, Reginald K. Lee, Amnon Yariv, Life Fellow, IEEE, and Axel Scherer
}

\begin{abstract}
We show that by placing a slab of semiconductor material between two photonic bandgap (PBG) mirrors, waveguide modes at frequencies out of the PBG can be obtained. These modes are similar to the modes of a conventional dielectric slab waveguide. Using these modes, we can obtain very good coupling between a PBG waveguide and a dielectric slab waveguide with similar slab properties. We discuss the properties of these slab modes and outline the guideline for the optimization of the PBG waveguides based on these properties.
\end{abstract}

Index Terms-Dielectric loaded waveguides, distributed Bragg reflection (DBR), optical coupling, periodic structures, photonic bandgap (PBG), photonic crystals, waveguides.

\section{INTRODUCTION}

$\mathbf{P}$ HOTONIC crystals [1], [2] have inspired a lot of interest recently due to their potential for controlling the propagation of light. Photonic crystals with line defects can be used for guiding light. Waveguides using one-dimensional (1-D) periodic structures were proposed by Yeh et al. [3]. Guiding light through sharp bends has been recently proposed [4] and demonstrated at microwave [5] and optical [6], [7] frequencies. Several aspects of the photonic crystal waveguides have been investigated [8]-[15]. In all the proposals and demonstrations of photonic bandgap (PBG) waveguides, the guiding modes were restricted to the PBG of the infinite photonic crystal. Furthermore, the PBG waveguides in the previous reports [4]-[6] were made by removing one row of either air columns or dielectric rods that results in multimode guiding. Single mode waveguides, however, are more desirable for practical applications. We recently studied [16] a more general type of PBG waveguide which consists of a dielectric slab placed between two PBG mirrors as shown in Fig. 1. Such a PBG waveguide can be made single mode by choosing the appropriate thickness ( $d$ in Fig. 1) for the dielectric slab. We further showed that there exist guiding modes in a PBG waveguide with very similar properties to the modes of a conventional dielectric waveguide. Such PBG waveguide modes, which we refer to as slab modes, exist even outside the photonic band gap of the crystal. The similarity between the fundamental slab mode of a PBG waveguide and that of a dielectric slab waveguide results in efficient coupling of power from the slab waveguide to the PBG waveguide over a wide frequency range.

Manuscript received May 23, 2000; revised July 18, 2000. This work was supported in part by the Air Force Office of Scientific Research under Contract AFOSR-61557, and by the Office of Naval Research (Y. S. Park).

A. Adibi is with the Georgia Institute of Technology, School of Electrical and Computer Engineering, Atlanta, GA 30332 USA.

Y. Xu, R. K. Lee, A. Yariv, and A. Scherer are with the Departments of Electrical Engineering and Applied Physics, California Institute of Technology, Pasadena, CA 91125 USA.

Publisher Item Identifier S 0733-8724(00)09826-1.
Two important physical mechanisms for guiding light in PBG waveguides are total internal reflection (due to the index contrast between middle slab and cladding) and Bragg reflection (due to the presence of photonic crystal). Similar mechanisms are responsible for guiding in photonic crystal fibers [17], which have a periodic array of holes. These photonic crystal fibers come in two varieties: 1) fibers whose symmetry has been broken by replacing the central hole with glass to create a high index defect and 2) fibers whose symmetry has been broken by replacing the central hole with a larger hole. In the former case, guiding is achieved by total internal reflection as the average index in the dielectric region with the holes (the cladding region) is less than that in the central filled region (or core region) [18].

Although the general guiding mechanisms in planar PBG waveguides and photonic crystal fibers are similar, there are two major differences between the two types of waveguides. First, the plane of discrete periodicity of the two-dimensional (2-D) photonic crystal is perpendicular to the guiding direction in photonic crystal fibers while it includes the direction of propagation in planar PBG waveguides. In other words, planar PBG waveguides have discrete periodicity in the guiding direction while photonic crystal fibers do not. As a result, distributed Bragg reflection (DBR) in the guiding direction is always present in PBG waveguides while it is not present in photonic crystal fibers.

The second difference between planar PBG waveguides and photonic crystal fibers is the confinement of light in air-core structures. Light is well confined in the core of an air-core photonic crystal fiber, since the plane of 2-D periodicity of the photonic crystal is perpendicular to the direction of propagation. On the other hand, the photonic crystal in a planar PBG waveguide can only help confine the light in one perpendicular direction (compared to the guiding direction), since the plane of periodicity includes the propagation direction. In the numerical analysis, we assume the third dimension to be infinite. However, this assumption is not valid in fabricating actual structures. Therefore, the light can not be confined in the third direction in air-core PBG waveguides. In dielectric-core PBG waveguides, the confinement in the third direction is provided by total internal reflection. This explains why previous experimental work in planar photonic crystal optical waveguides has been performed using dielectric-core waveguides. Therefore, we limit our attention in this paper to dielectric-core photonic crystal optical waveguides.

In this paper, we show that the properties of the fundamental mode of a PBG waveguide are mainly affected by the total internal reflection (similar to a conventional dielectric slab waveguide) and the distributed Bragg reflection (due to the presence 
of the photonic crystal). We also show that the range of loss-less guiding in a PBG waveguide is not limited to the PBG. In other words, the presence of an absolute PBG is not necessary for loss-less guiding. The main limitation for the loss-less guiding in a PBG waveguide is the coupling of the guided mode to other modes of the photonic crystal that are extended to the photonic crystal region. The absence of photonic crystal modes with the same frequency and wavevector as those of the guided mode are necessary for loss-less guiding. Therefore, the main role of PBG in dielectric-core PBG waveguides is to provide a frequency range in which there is no other mode to couple the guided mode into. The actual frequency range with this property is usually bigger than the absolute PBG. Having this fact in mind, we use the phrase "PBG waveguide" to refer to the waveguides made by generating a line defect in a photonic crystal without implying that the guiding mechanism is due to the PBG itself.

Understanding the properties of the PBG waveguides is important in explaining the experimental results, and designing PBG waveguides appropriately. For example, some experimental results for the design of bends using photonic crystal optical waveguides have been recently reported. In explaining these experimental results, it has been assumed that the guiding in a PBG waveguide is limited to the PBG. In this paper, we show that this assumption is not necessarily true. Therefore, the low experimental transmission through the bends in the reported experiments can be due to the guided modes out of the PBG that are not efficiently transmitted in waveguide bends.

Efficient coupling of light into and out of a PBG waveguide (for example, from an optical fiber) is important in both performing experiments with PBG waveguides and in designing optical elements using photonic crystals. We show in this paper that a dielectric slab waveguide can be coupled efficiently in a wide frequency range to a PBG waveguide and vice versa. Methods for efficient coupling of light into and out of a dielectric slab waveguide (from a fiber or from free space) are well known. For example, tapered slab waveguides can be used for optimizing the coupling efficiency. Therefore, our results suggest that we can use dielectric slab waveguides for coupling light into and out of PBG waveguides as well as for connecting optical elements made from photonic crystals.

In this paper, we discuss the properties of the slab modes of a PBG waveguide in detail. We also present the numerical analysis of the coupling of a dielectric slab waveguide to a PBG waveguide. We discuss the numerical method we used in our simulations in Section II. The properties of the fundamental slab mode of a PBG waveguide are discussed in Section III. We discuss the different issues in coupling of a dielectric slab waveguide to a PBG waveguide in Section IV. The importance of the slab modes of a PBG waveguide and some suggestions about the applicability of the results of this paper are discussed in Section V, and final conclusions are drawn in Section VI.

\section{Simulations}

The main structure we used in the analysis of this paper is the connection of a dielectric slab waveguide to a PBG waveguide as shown in Fig. 1. The thicknesses of the middle slabs of both waveguides are equal to $d$. The photonic crystal we

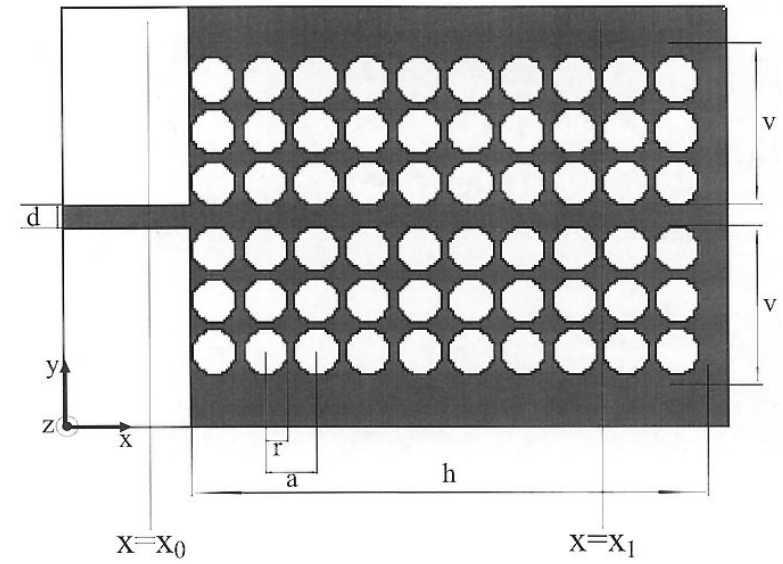

Fig. 1. Connection of a dielectric slab waveguide to a PBG waveguide. The PBG waveguide is made by putting a dielectric slab of thickness $d$ between two PBG mirrors. Each PBG mirror is composed of $v / a$ periods of a square lattice of air columns in the same dielectric as the middle slab. The length of the PBG waveguide is assumed to be $h$. The period of the photonic crystal and the radius of the air columns are equal to $a$ and $r$, respectively. For all structures analyzed in this paper, $a=24$ calculation cells and $r / a=0.45$ are used. Furthermore, perfectly matched layers are used around the structure (left, right, top, and bottom). We also put a thin layer (ten calculation cells) of either dielectric or air between the PBG and PML on top and bottom of the structure. The dielectric used in this paper is GaAs $(\epsilon=12.96)$.

use throughout this paper is a 2-D square lattice of infinite air columns in gallium arsenide (GaAs) as shown in Fig. 1. The radius of the air holes $(r)$ is $45 \%$ of the lattice period $(a)$ to assure the existence of a PBG for TE modes (electric field parallel to the axis of the air columns). The length of the slab waveguide is twice the period length $(a)$ of the photonic crystal, while the length of the PBG waveguide is shown by $h$. The thickness of each PBG mirror in the vertical dimension is shown by $v$. To analyze the guiding structures, we used a computer code based on the 2-D finite difference time domain (2D-FDTD) method [19]. We also put perfectly matched layers (PML) [20] around the structures at all boundaries. Finally, we assume that there exists a thin layer of either air or GaAs between the PBG mirror and the PML on top and bottom of the structure. In the calculations of this paper, the speed of light in vacuum $(c)$ is normalized to one, and all spatial dimensions are in the units of calculation cells.

To calculate the power transmission spectrum (transmission coefficient versus frequency) from the slab waveguide to the PBG waveguide, we used a pulsed Huygens source [21] to excite the fundamental TE mode in the slab waveguide at $x=x_{0}$ in Fig. 1. We calculated the spectrum of the power transmitted through the PBG waveguide by taking the Fourier transform of the fields and then integrating the Poynting vector over a surface of 60 calculation cells centered at the middle of the PBG slab at $x=x_{1}$ in Fig. 1. The power transmission coefficient was then calculated as the ratio of the transmitted power to the incident power. The field patterns in the structure of Fig. 1 were calculated using a constant frequency Huygens source to excite the fundamental TE mode (electric field normal to the calculation plane) in the slab waveguide at $x=x_{0}$.

To calculate the dispersion diagram of the slab mode of the PBG waveguide, we used an order-N spectral method [22] in the computational domain shown in Fig. 2(b) with Bloch boundary 


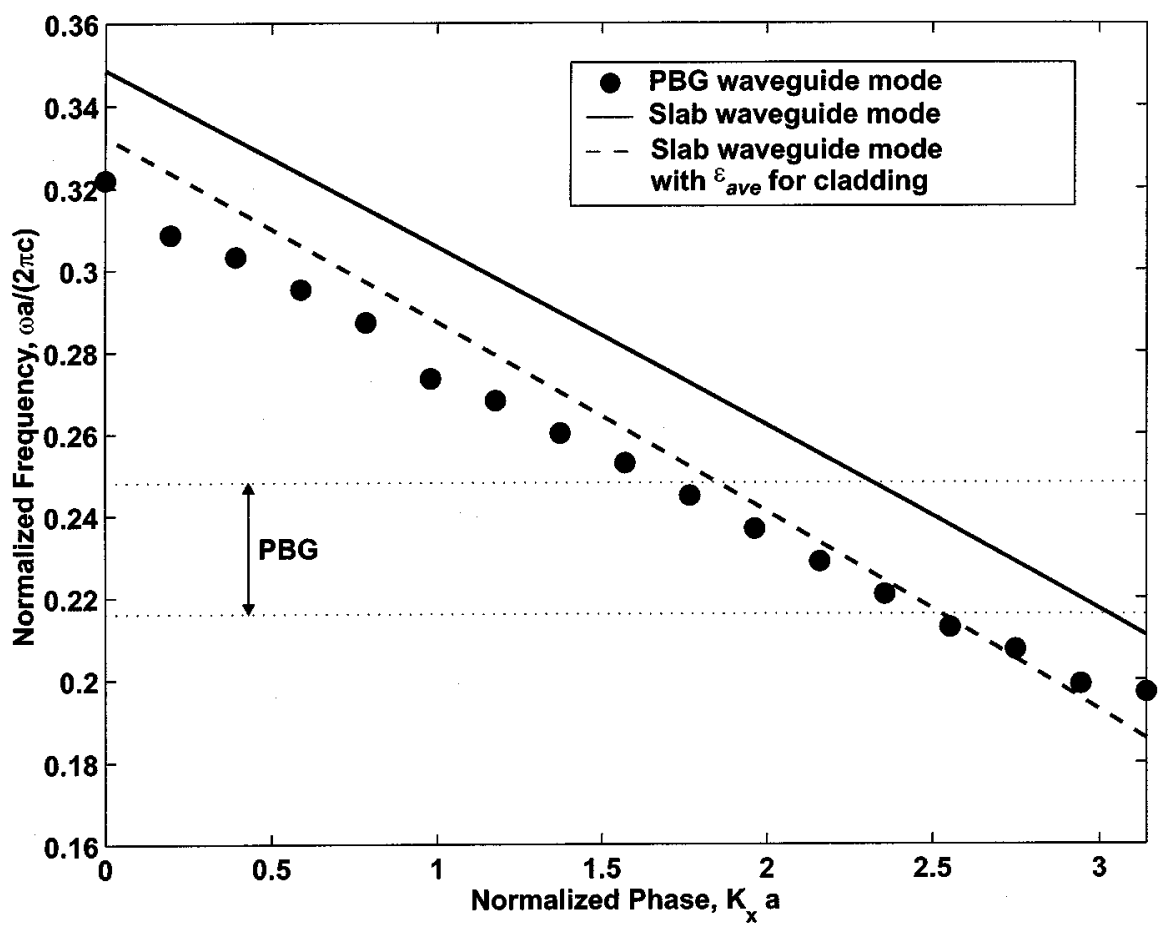

(a)

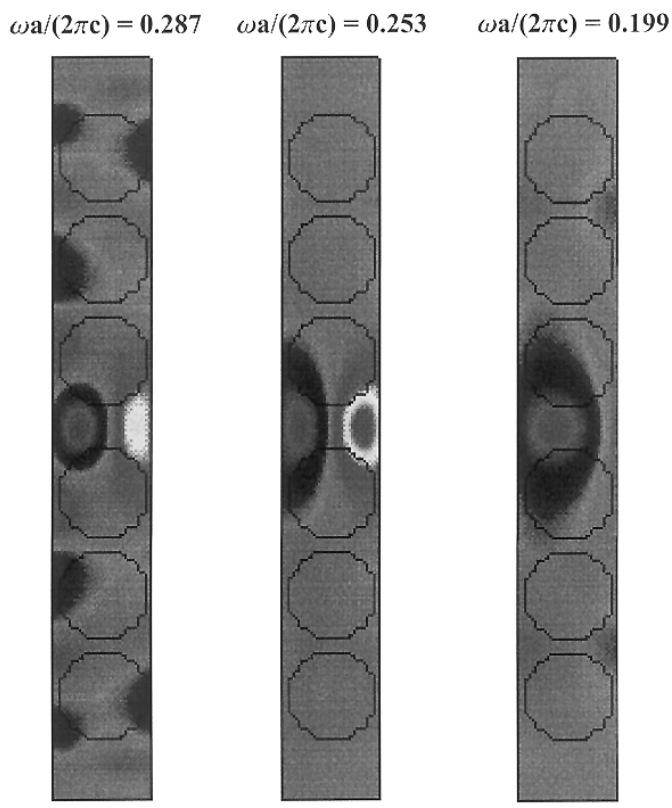

(b)

Fig. 2. Properties of the PBG waveguide mode. (a) Dispersion diagram of the modes of a PBG waveguide and a dielectric slab waveguide with equal slab thicknesses $(d=9$ calculation cells $)$ and permittivities $(\epsilon=12.96)$. The cladding region of the slab waveguide is either air $(\epsilon=1)$ or a material with permittivity $\epsilon_{\text {ave }}=5.35$ equal to the average $\epsilon$ of the PBG mirrors in the PBG waveguide. The PBG waveguide has the same specifications as in the caption of Fig. 1 with a thin (ten calculation cells) GaAs on top and bottom of the PBG mirrors. The bandgap of the photonic crystal covers from $\omega a /(2 \pi c)=0.216$ to $\omega a /(2 \pi c)=0.248$ as shown by the dotted lines. (b) Electric field pattern of the TE mode (electric field parallel to axis of the air columns) in the PBG waveguide at different frequencies.

conditions on the left and right sides and perfectly matched layers (PML) on the top and bottom of the structure. The electric field patterns of the slab mode of the PBG waveguide at different frequencies were obtained through similar procedure by exciting the structure with a constant frequency source. More details of our numerical algorithms can be found in [23] and [24].

\section{Slab Modes IN PBG Waveguides}

Fig. 2(a) shows the dispersion diagram for the TE mode of the PBG waveguide shown in Fig. 1. The parameters of the PBG waveguide used in this calculation are $d=9$ and $v=3 a$ with $a$ being the period of the photonic crystal. Fig. 2(b) shows the electric field pattern of this mode at three different frequencies. 
The mode shown in Fig. 2 is a confined mode with its energy concentrated mainly in the middle slab region at the frequencies in the middle of the dispersion band [Fig. 2(a)]. The mode is lossy at both edges of the dispersion band $(\omega<0.204$ and $\omega>0.267)$. We have also shown the dispersion curves of the fundamental TE mode (with electric field normal to the calculation plane) of a conventional dielectric slab waveguides with the same slab thickness $(d=9)$ and permittivity $\left(\epsilon_{1}=12.96\right)$ as the middle slab of the PBG waveguide. The region above and below the slab in the dielectric slab waveguides is assumed to be air $\left(\epsilon_{2}=1\right)$. The dispersion curve of the slab waveguide mode in Fig. 2(a) is restricted to the first Brillouin zone of the slab waveguide with imposed artificial spatial periodicity $a$, the same as that of the photonic crystal waveguide.

As Fig. 2(a) shows, the PBG waveguide mode has a similar dispersion diagram to the slab waveguide mode. The dispersion curve of the TE slab mode of the PBG waveguide becomes more similar to that of the fundamental mode of a slab waveguide with $d=9$ if we replace the air cladding regions $\left(\epsilon_{2}=1\right)$ with a material with average permittivity $\epsilon_{\text {ave }}=\epsilon_{1}-\pi(r / a)^{2}\left(\epsilon_{1}-\epsilon_{2}\right)=$ 5.35 as shown in Fig. 2(a). The difference between the dispersion diagrams of the two modes is due to the higher confinement of the PBG waveguide mode that is the result of the presence of the PBG mirrors (instead of a continuous dielectric) on both sides of the middle slab. The relatively flat behavior of the dispersion diagram of the slab mode of the PBG waveguide at the high symmetry point $X\left(K_{\mathrm{X}} a=\pi\right)$ is due to the DBR from the periodic structures on the top and bottom of the slab region. The main difference between the PBG waveguide mode and the slab waveguide mode is that the former has some loss at low and high frequencies due to the coupling of energy to the modes that propagate in the photonic crystal at frequencies outside the PBG. This loss is negligible for a range of frequencies $(0.204<\omega a /(2 \pi c)<0.267)$ that is larger than the PBG $(0.216 \leq \omega a /(2 \pi c) \leq 0.248)$. As Fig. 2(b) suggests, the field pattern of the PBG waveguide mode is similar to that of the slab waveguide. Therefore, we expect very good coupling between the two waveguides over a relatively wide frequency range.

\section{Coupling of a Slab Waveguide to a PBG WaVeguide}

The spatial similarities between the slab modes of a PBG waveguide and those of a dielectric slab waveguide suggest that we can expect efficient power transmission from one waveguide to the other. Fig. 3 shows the electric field pattern of a TE mode (electric field parallel to the axis of the air columns) at the intersection between a slab waveguide and a PBG waveguide with similar slab thicknesses and permittivities. The frequency of the source used in this calculation was $\omega a /(2 \pi c)=0.258$, which is out of the bandgap. The PBG waveguide mode at this frequency (even though it is out of the PBG) has very low loss since it is well confined in the slab region.

Fig. 4 shows the power transmission coefficient (from the slab waveguide to the PBG waveguide) versus frequency for the waveguide connection shown in Fig. 3. As Fig. 4 shows, it is possible to have a high transmission coefficient between a slab waveguide and a PBG waveguide over a relatively wide range of frequencies (larger than the $\mathrm{PBG}$ ) corresponding to the

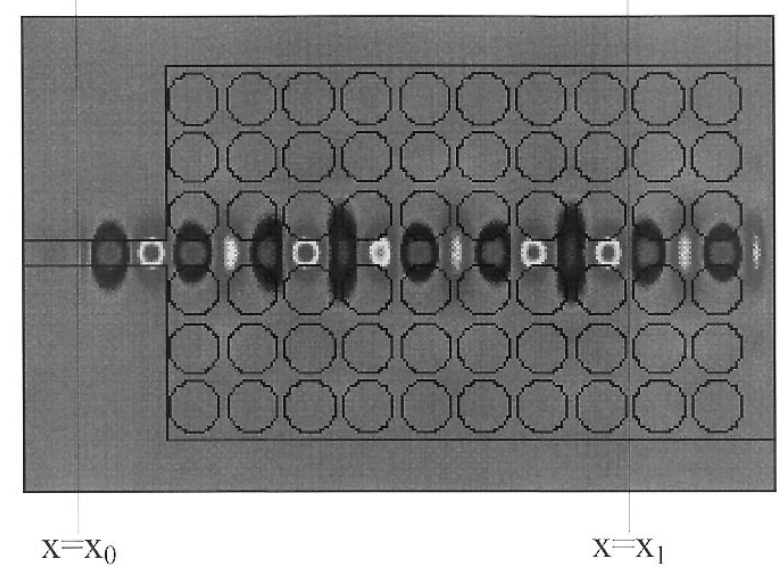

Fig. 3. Electric field pattern of the TE mode (electric field parallel to axis of the air columns) for the intersection of a dielectric slab waveguide and a PBG waveguide with equal slab thicknesses. The specifications of the waveguides are the same as those in Fig. 1. The normalized frequency of the source is $\omega a /(2 \pi c)=0.258$, which is out of the PBG of the photonic crystal.

range of frequencies over which the PBG waveguide mode has very low loss. The transmission coefficient at higher frequencies $(\omega a /(2 \pi c)>0.28)$ is smaller due to the excitation of other modes of the PBG structure that are lossy. These modes attenuate as they propagate along the PBG waveguide. As Fig. 4 shows, putting air instead of GaAs on top and bottom of the entire PBG waveguide (between the PBG mirrors and the PML) will affect the smaller peak [at $\omega a /(2 \pi c) \simeq 0.295$ ] in the transmission coefficient. However, the high transmission behavior at middle frequencies is not affected considerably by the material at the top and bottom of the structure. This is due to the fact that for these frequencies, the mode is well confined and is not influenced by the structure far from the slab region.

To understand the different aspects of the coupling between a slab waveguide and a PBG waveguide, we need to investigate the effects of the design parameters in the power transmission spectrum. The effect of the top and bottom medium (air or semiconductor) was discussed above. In the following, we discuss the effects of the horizontal and vertical sizes of the PBG waveguide as well as the effect of the slab size.

\section{A. Effect of the Length of the PBG Waveguide ( $h$ )}

A very interesting phenomenon in the coupling of a slab waveguide to a PBG waveguide is DBR [25]. This effect depends on the length of the periodic structure in the guiding direction. Fig. 5(a) shows spectra of power transmission from a slab waveguide to two PBG waveguides with different lengths ( $h$ in Fig. 1). The power reflection spectra for the two structures are shown in Fig. 5(b). We included a lower frequency range in the spectra shown in Fig. 5(a) and (b) to show the reflection peak (or the transmission minimum) due to the DBR effect at $\omega=0.180$. The thicknesses of the PBG mirrors on the top and bottom of the middle slab for the two cases was the same $(v=3 a)$. Fig. 5(b) shows that the reflection peak is stronger for the longer PBG waveguide $(h=10 a)$ due to the longer interaction region between the electric field and the periodic structure. The main reflection peak and the subsequent smaller 


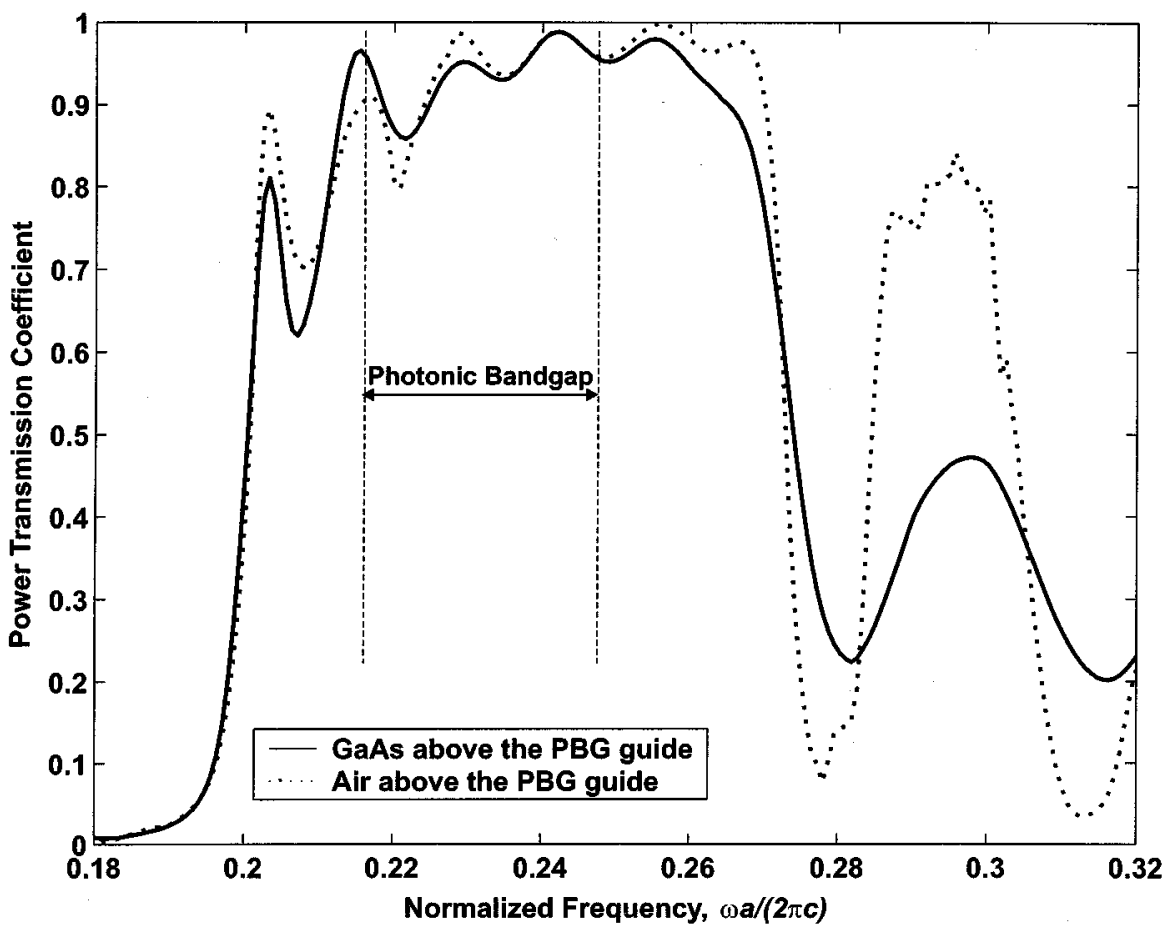

Fig. 4. Spectrum of power transmission coefficient from a dielectric slab waveguide to a PBG waveguide with similar slab properties. The solid curve and the dotted curve show the power transmission coefficients for the cases where there is a GaAs or an air buffer layer on top and bottom of the PBG mirrors (between the PBG and the PML), respectively. The thicknesses of both buffer layers are ten calculation cells.

reflection peaks for the longer waveguide $(h=10 a)$ are narrower than those of the shorter waveguide $(h=6 a)$.

Fig. 5 shows that the variation of the power transmission coefficient within the high transmission region $(0.206<\omega<270)$ is mainly due to the DBR effect, and not due to the power loss from energy coupling to other PBG modes. This argument also explains why the longer waveguide has faster variation (or more local maxima and minima) of the transmission spectrum in the high transmission frequency window compared to the shorter waveguide. If these variations were due to the waveguide loss, the power transmission coefficient would be much smaller than what we calculated for the longer waveguide (as a result of exponential power loss). Note also that for PBG waveguides longer than some minimum length, the effect of the DBR does not significantly change with the length of the waveguide.

\section{B. Effect of the PBG Mirror Thickness (v)}

Fig. 6 shows the transmission spectra from a slab waveguide to two PBG waveguides with different PBG mirror thicknesses ( $v$ in Fig. 1). Both PBG waveguides used in this calculation had the same length $(h=6 a)$. Other properties of the structures are mentioned in the caption. As Fig. 6 shows, the power transmission coefficient over the high transmission window $(0.206<$ $\omega<0.270$ ) is not highly influenced by changing the thickness of the PBG mirrors $(v)$. This is another indication that the electromagnetic energy in this frequency window is highly concentrated in the slab region.

We attribute the small variation of the power transmission coefficient with $v$ in the high transmission window to the excitation of the surface modes at the intersection of the two waveguides. To support this argument, we have shown the electric field patterns of the fundamental TE mode at $\omega=0.23$ in the two structures with different PBG mirror thicknesses $(v)$ in Fig. 7(a) and (b). Fig. 7 (b) shows stronger excitation of the surface modes in the structure with thicker PBG mirror $(v=8 a)$ at this frequency. We can think of these surface modes as the modes of a Fabry-Perot structure placed between the slab and the outside (air) region. The frequencies of these surface modes are different for different PBG mirror thicknesses $(v)$. Therefore, the small local minima of the transmission spectra in the high transmission window corresponding to these surface modes occur at different frequencies. Furthermore, the number of the surface modes as well as the power coupling from the guiding mode into these surface modes are larger in the thicker structures resulting in more variations of the transmission coefficient in the high transmission window. It is important to note that the reduction of the transmission power due to the excitation of the surface modes is much smaller than that due to the DBR effect.

\section{Effect of the Middle Slab Size $(d)$}

Fig. 8 shows the power transmission spectra from a slab waveguide to a PBG waveguide for three different slab sizes ( $d$ in Fig. 1) of the two waveguides. As Fig. 8 shows, the frequency of the minimum transmission due to the DBR effect becomes smaller as we increase the slab thickness $(d)$. This can be explained by the following argument: The field strengths in the dielectric and air regions of a structure with larger $d$ at any frequency $\omega_{1}$ are similar to those of a structure with smaller $d$ at a higher frequency $\omega_{2}>\omega_{1}$ (or a shorter wavelength). This is due to the scaling properties of the Maxwell's equations [26]. Therefore, we expect the peaks of the DBR effect to be 


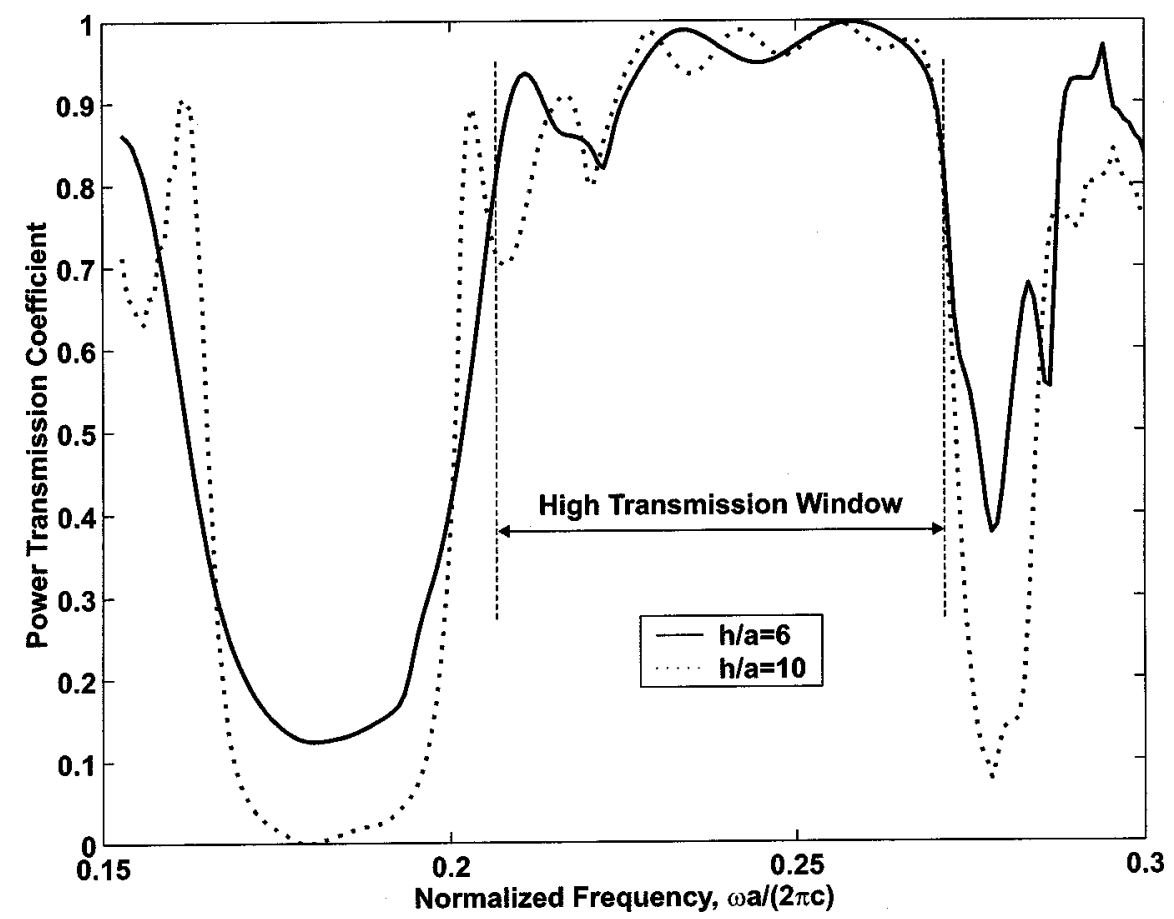

(a)

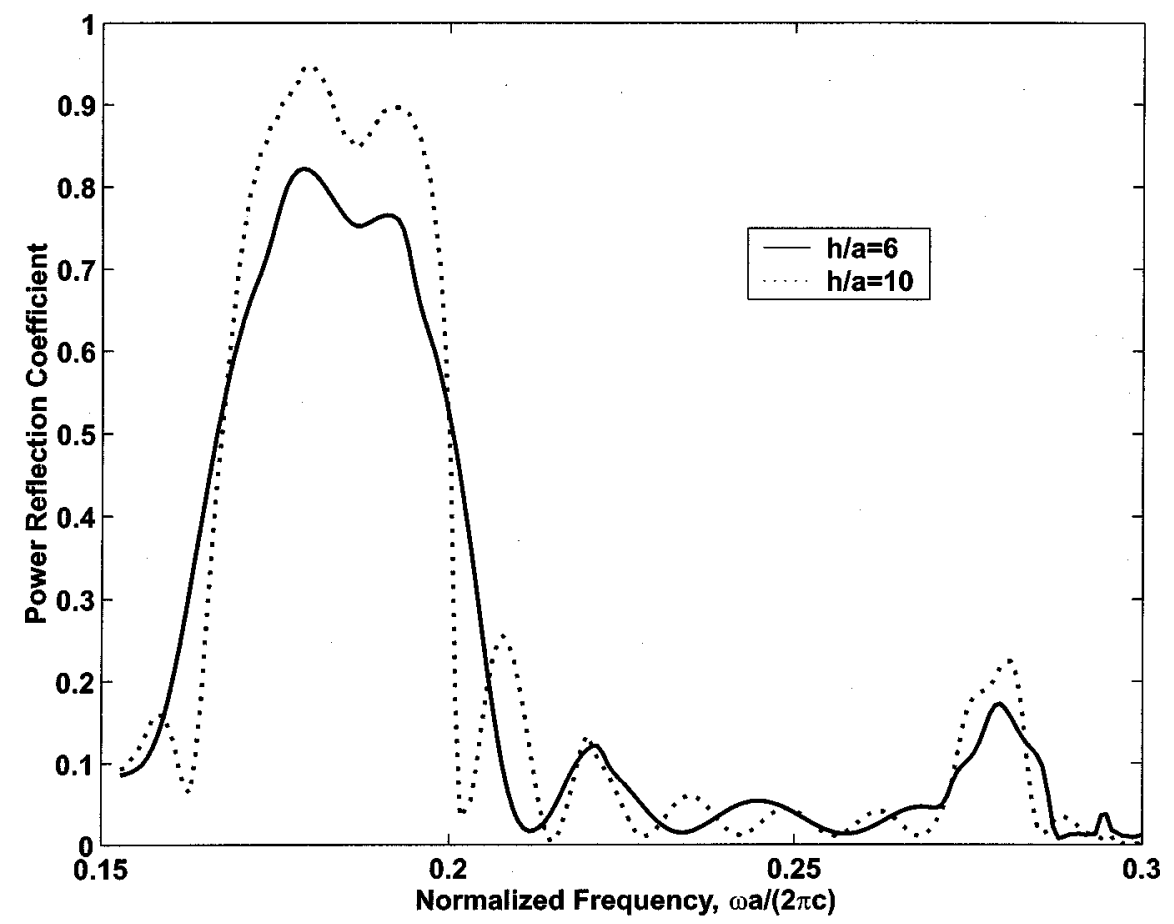

(b)

Fig. 5. Effect of the PBG waveguide length on the coupling of a slab waveguide to a PBG waveguide. (a) Transmission and (b) reflection spectra from the slab waveguide to two different PBG waveguides with lengths $h=6 a$ and $h=10 a$. The thicknesses of the slab and the PBG mirrors in both cases are $d=9$ and $v=3 a$, respectively. The media between the PBG mirror and the PML on the top and bottom of the structure are air in both cases. The excitation source is placed at $x_{0}=40$, and the reflection spectra are calculated at $x=20$ in both cases. The transmission spectra are calculated at $x_{1}=155$ and $x_{1}=251$ for the shorter and longer waveguides, respectively.

shifted to lower frequencies as we increase the slab thickness $d$. It is important to note that the position of a DBR peak does not inversely scale with $d$ (as one might expect) since the photonic crystal regions on top and bottom of the slab are the same (and not scaled with $d$ ) in these calculations. We should also note the coupling of the slab mode with the other modes of the photonic crystal at higher frequencies. As $d$ becomes larger, the DBR peak at higher frequencies $(\omega>0.25)$ moves toward lower frequencies where the coupling to the other modes of the photonic crystal is reduced. Therefore, the power loss due to the coupling to these extra modes is reduced, and higher DBR peaks are obtained. 


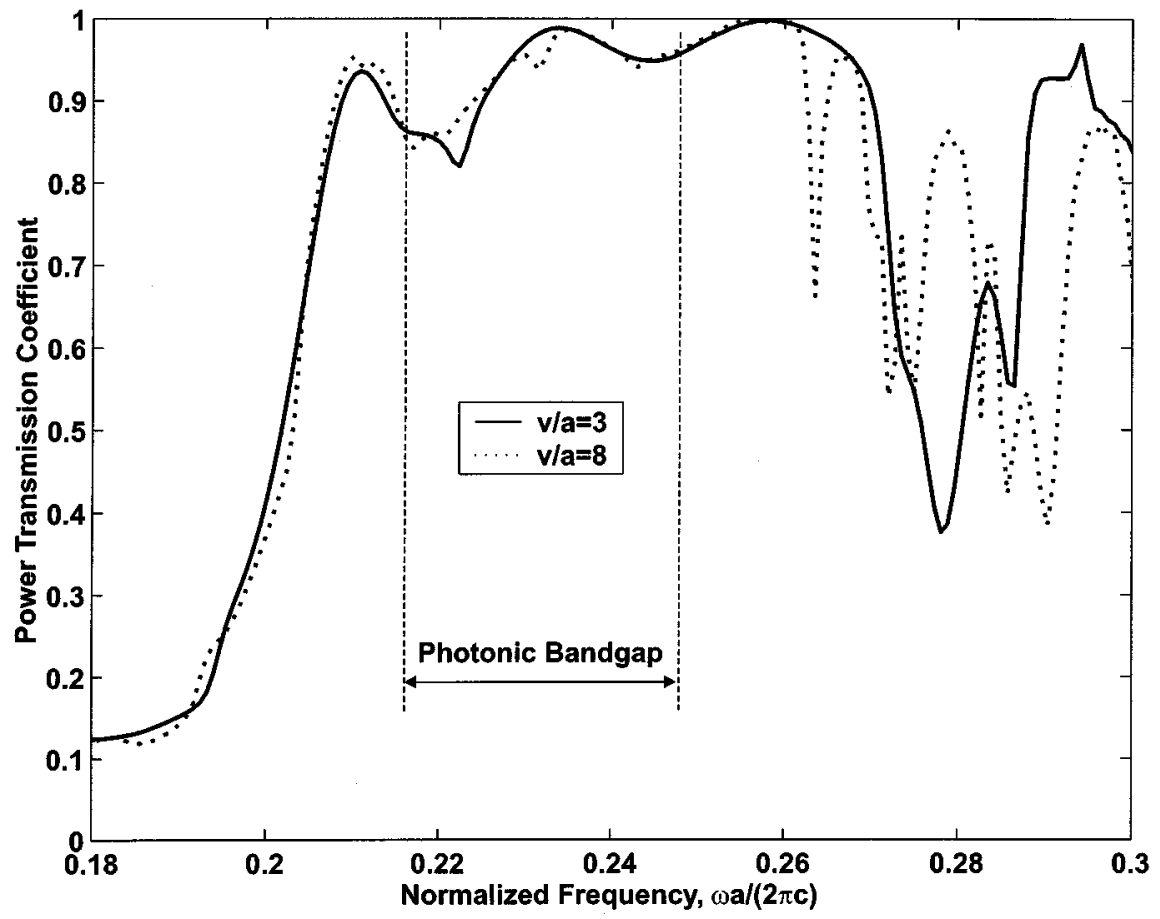

Fig. 6. Transmission spectra from a slab waveguides to two PBG waveguide with different PBG mirror thicknesses $v=3 a$ and $v=8 a$. Other parameters in both cases are $d=9, h=6 a, x_{0}=40$, and $x_{1}=155$. The media between the PBG mirrors and the PMLs on the top and bottom of the structure are air in both cases.

a

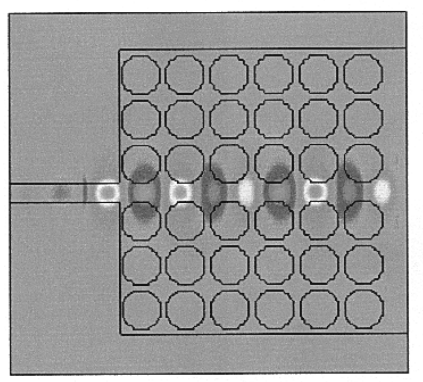

b

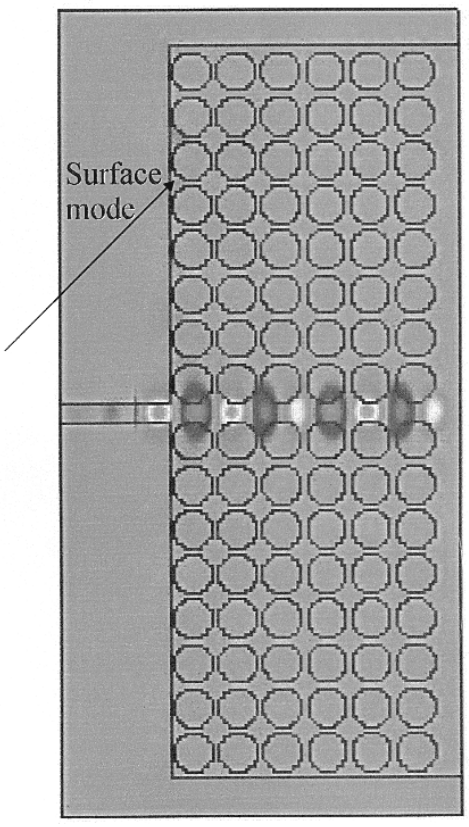

Fig. 7. TE electric field pattern at $\omega=0.23$ at the intersection of a slab waveguide and a PBG waveguide with (a) $v=3 a$, and (b) $v=8 a$. Other parameters in both cases are $d=9$ and $h=6 a$. The media between the PBG mirrors and the PMLs on the top and bottom of the structure are air in both cases. The excitation of the surface modes along the left interface of the photonic crystal in part (b) is evident.

It is important to note that all the coupling aspects discussed here are not limited by the photonic band gap of the crystal. The important frequency ranges to consider are where extra (or extended) modes of the photonic crystal exist, and where DBR peaks occur. For example, we can have low power transmission from the slab waveguide to the PBG waveguide even within the bandgap if the DBR effect has a peak there.

\section{Coupling Out of the High Transmission Window}

Although we are mainly interested in the range of frequencies where we can obtain efficient transmission from the slab waveguide to the PBG waveguide, it is instructive to investigate the power transmission spectrum at higher frequencies. Fig. 9 shows the power transmission spectra for different PBG mirror thicknesses $(v)$ at these frequencies. The loss in power 


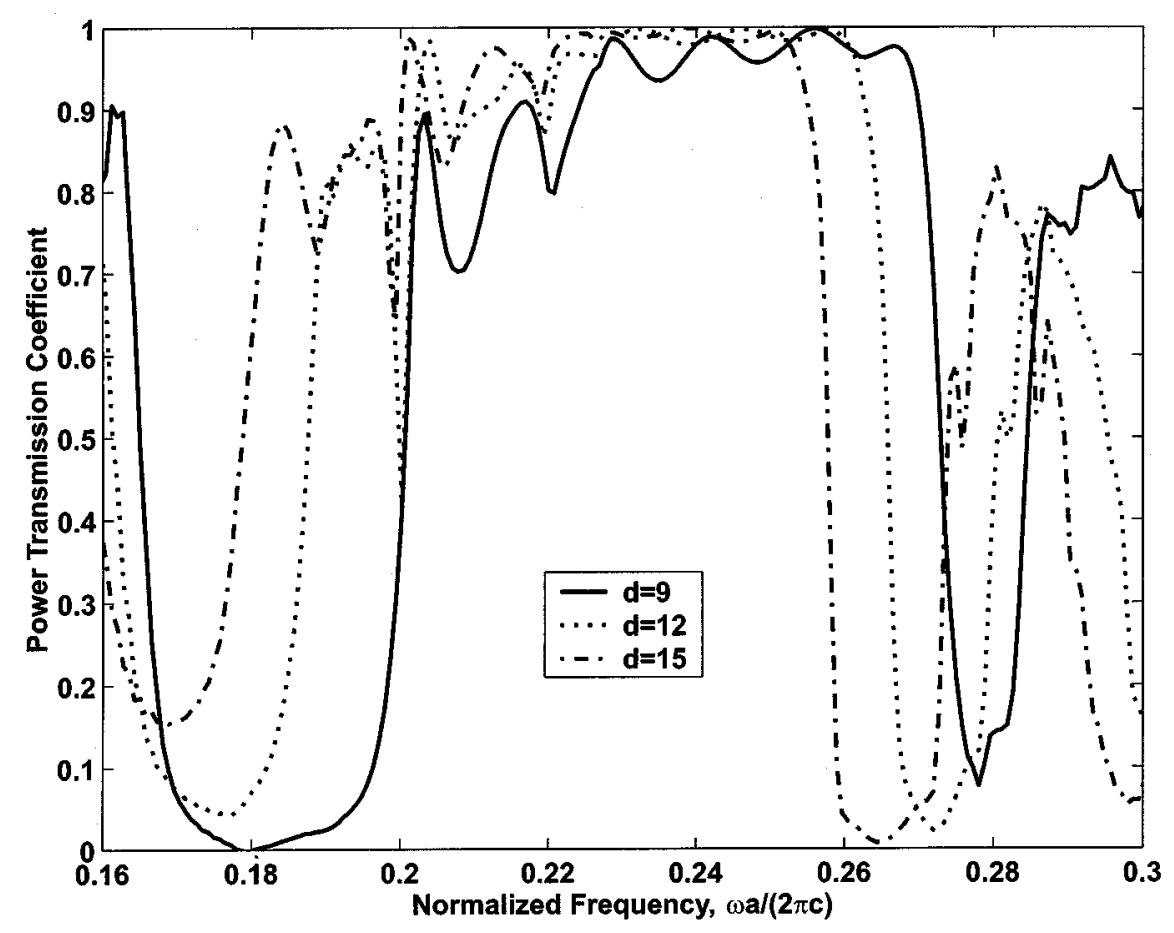

Fig. 8. Effect of the slab thickness $(d)$ on the coupling of a slab waveguide to a PBG waveguide. Transmission spectra from the slab waveguide to three different PBG waveguides with $d=9, d=12$, and $d=15$. Other parameters in all cases are $h=10 a, v=3 a, x_{0}=40$, and $x_{1}=251$. The media between the PBG mirrors and the PMLs on the top and bottom of the structure are air in all cases.

transmission at higher frequencies is mainly due to the coupling of energy from the slab mode to the other modes of the photonic crystal. Therefore, the electromagnetic energy is more distributed inside the structure, and the wave propagation in the PBG waveguide is affected by the structure even far from the slab region. Fig. 9 shows that the number of transmission minima in the higher frequency regime is larger for larger thickness of the photonic crystal $(v)$ on the top and bottom of the middle slab. This behavior is similar to the power transmission through a Fabry-Perot structure. Fig. 10 shows the electric field pattern of the TE mode of the structure at $\omega=0.302$. The air regions between the PBG mirrors and the PMLs in the calculation of Fig. 10 are extended to show the radiation of the power out of the structure. The power loss due to the coupling of energy to the modes of the photonic crystal is visible from Fig. 10. It is also important to note that the DBR effect still exists at high frequencies. However, the reflection peaks are not as strong as they are at lower frequencies due to the power loss from coupling to the modes of the photonic crystal.

Although Fig. 9 shows some transmission peaks with more than $80 \%$ power transmission at higher frequencies, we can not use the PBG waveguide at these frequencies. The large peak transmissions in Fig. 9 is only due to the short length of the PBG waveguide used in our calculations. The waveguide modes at these frequencies are lossy and the transmitted power decays along the waveguide. The power loss is higher for longer waveguides due to higher power transfer to the modes of the photonic crystal. Therefore, the power transmission is reduced for longer waveguides. This is shown in Fig. 11, which shows the transmission spectra for the structures with different PBG waveguide lengths.

\section{E. Multimode Coupling of the Slab and the PBG Waveguides}

Until now, we concentrated mainly on the single-mode coupling of the slab and PBG waveguides. On the other hand, the PBG waveguides in the previous reports [4]-[6] were made by removing one row of either air columns or dielectric rods from the photonic crystal. The slab thickness in such waveguides is too thick for single-mode propagation. Although multimode waveguides are not desirable for practical applications, we briefly discuss the coupling of the slab and PBG waveguides in the multimode regime for the sake of completeness.

Fig. 12 shows the transmission spectrum from a slab waveguide to a PBG waveguide in the multimode regime excited with the fundamental mode of the dielectric slab waveguide. The PBG waveguide was made by removing one row of air holes resulting in the slab thickness of $d=27$ for both waveguides. The results shown in Fig. 12 are similar to those shown in Fig. 8 for larger $d$. As explained before, using a thick slab results in shifting the DBR peak to lower frequencies. Another effect in the multimode regime is that the larger confinement of the field in the slab region reduces the coupling of the slab mode to the other modes of the photonic crystal. This allows high transmission even in the higher frequencies where the modes of the photonic crystal exist. Other properties like the effects of the PBG mirror thickness and that of the guiding length are similar to those in the single-mode regime.

\section{DISCUSSION}

The results shown in this paper suggest that we can think of a PBG waveguide as the combination of a slab waveguide, a DBR, and a photonic crystal whose modes are responsible for power 


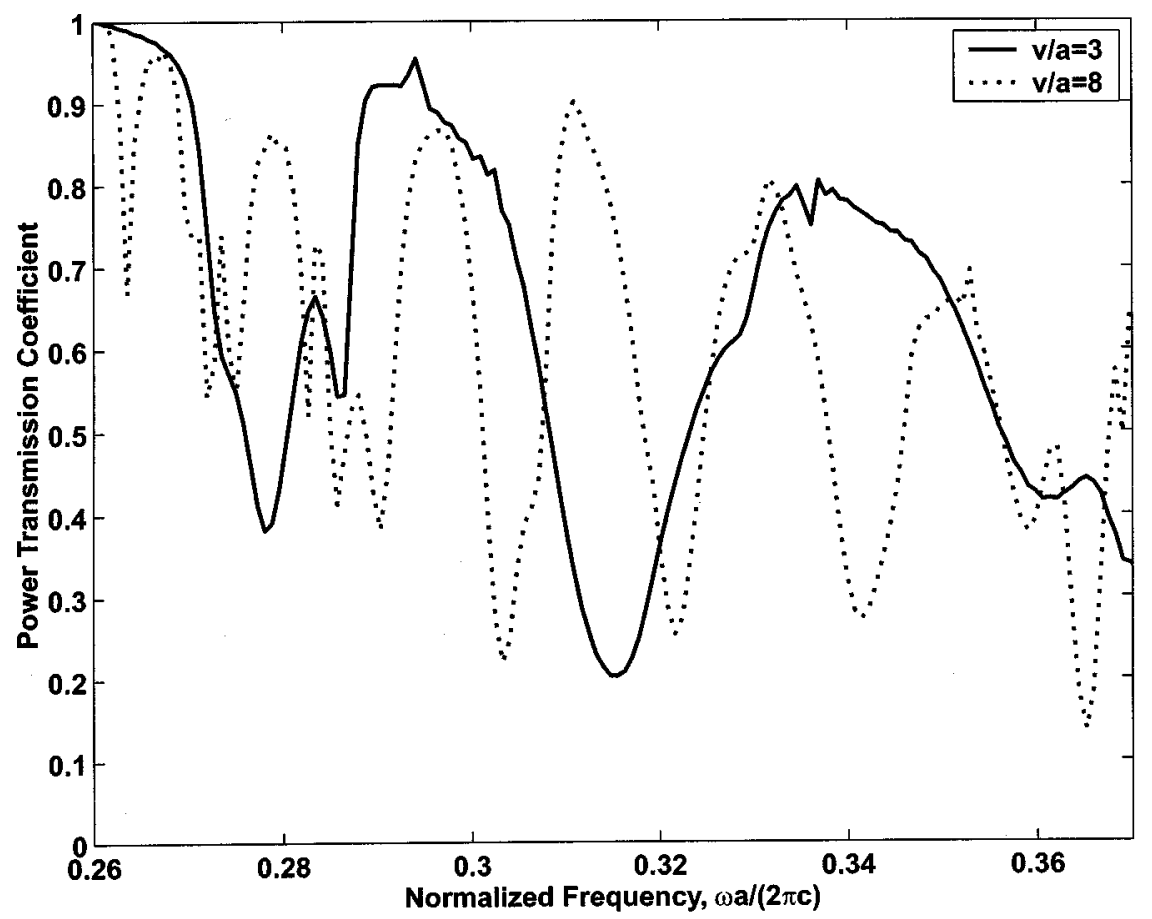

Fig. 9. Transmission spectra at higher frequencies from a slab waveguide to two PBG waveguide with different PBG mirror thicknesses $v$. The media between the PBG mirrors and the PMLs on the top and bottom of the structure are air. Other parameters are $d=9, h=10 a, x_{0}=40$, and $x_{1}=251$.

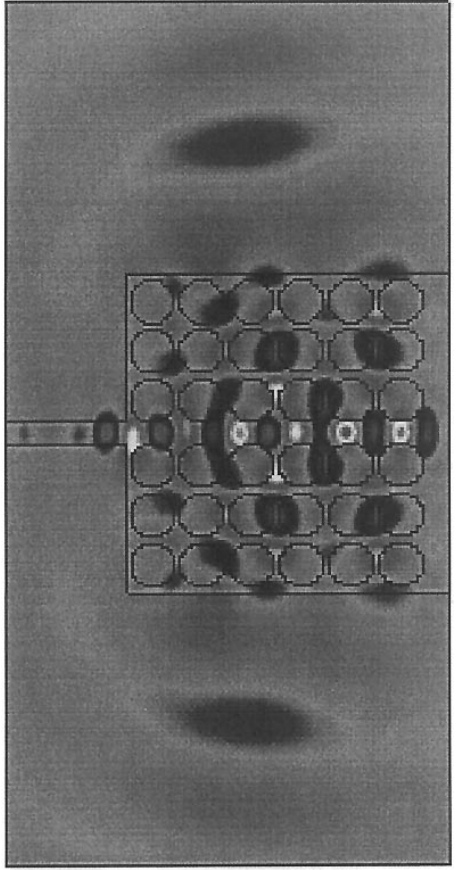

Fig. 10. TE electric field pattern at $\omega=0.302$ at the intersection of a slab waveguide and a PBG waveguide with $d=9, v=3 a$, and $h=6 a$. The media between the PBG mirrors and the PMLs on the top and bottom of the structure are air in both cases.

loss. These three portions of a PBG waveguide can be designed almost independently to obtain the desired performance. We can change the slab thickness easily. We can also change the properties of the DBR by changing the corrugations (geometrical deviations from a perfect flat slab caused by the photonic crystal) on the top and bottom of the slab. This can be done, for example, by changing the radii (and/or the centers, or even the shapes) of the air holes of the photonic crystal in the rows adjacent to (on top and bottom of) the middle slab. We can optimize the rest of the photonic crystal by choosing the lattice type, index contrast, and hole radii appropriately.

The slab modes are the key elements in understanding the efficient coupling of a slab waveguide to a PBG waveguide and vice versa. The major issues in the design of a high transmission window in the frequency domain are to make sure that the power is not coupled to any other PBG mode in that window, and to move the DBR peak frequencies far enough from the window. As a result, we can design the optical elements using photonic crystals, and use dielectric slab waveguides connected to short PBG waveguides to connect these optical elements. Our results also suggest that we can use dielectric slab waveguides for efficient coupling of light into and out of PBG waveguides through using the well-known methods for efficient coupling of light into and out of a dielectric slab waveguide (from a fiber or from free space). For example, a tapered slab waveguide can be used for optimizing the coupling efficiency.

We can also support surface modes in a dielectric slab by placing a PBG mirror on one side (top or bottom) of it. Again, the properties of the surface mode can be changed by changing the slab thickness, the corrugation at the slab surface, and the photonic crystal design.

The results shown in this paper prove the possibility of guiding in PBG waveguides out of the PBG. We believe that this fact needs to be considered in interpreting the experimental results regarding the PBG waveguides. In other words, we should not interpret all evidence of the guiding in PBG waveguides to the guided modes inside the PBG. 


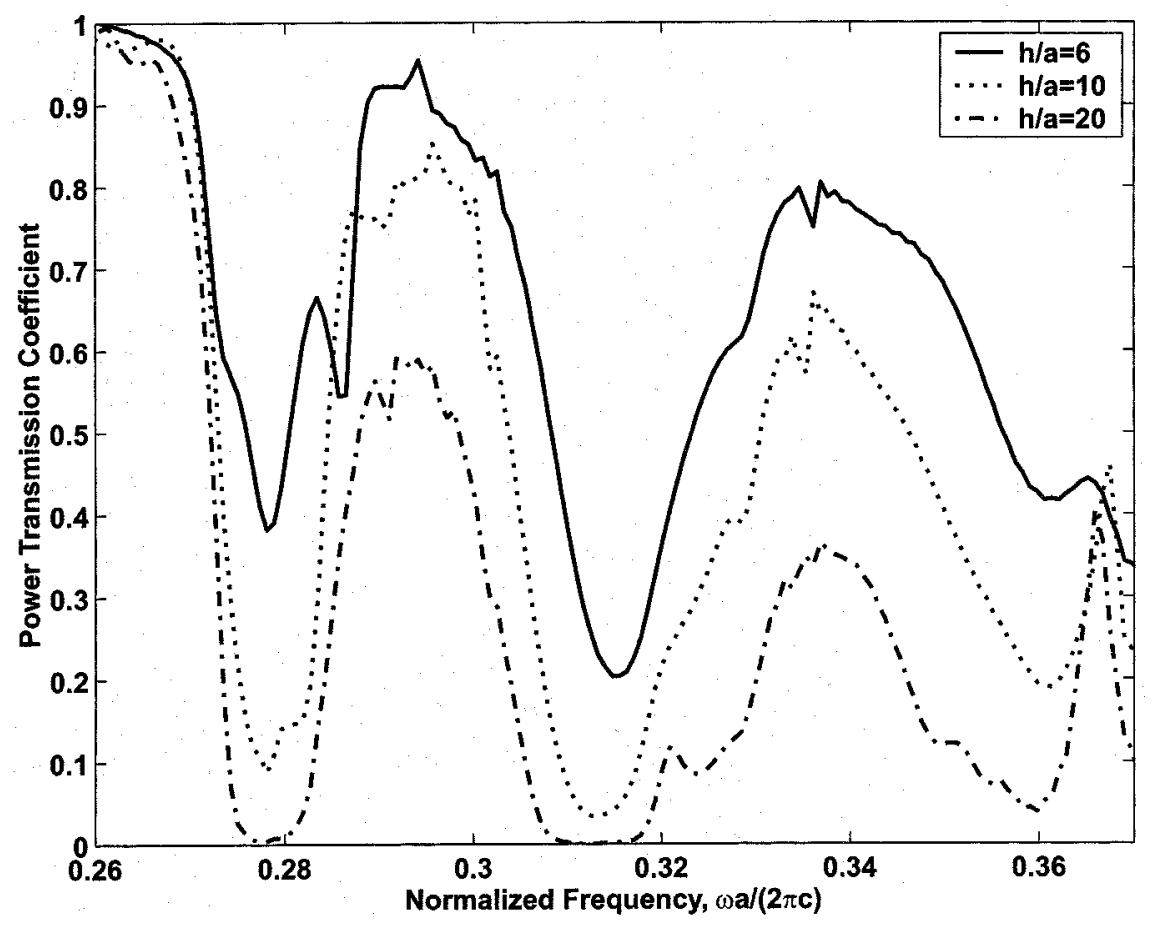

Fig. 11. Transmission spectra at higher frequencies from a slab waveguide to three PBG waveguide with different PBG waveguide length $d$. The thicknesses of the slab and the PBG mirrors are $d=9$ and $v=3 a$, respectively, and the media between the PBG mirrors and the PMLs on the top and bottom of the structure are air in all cases. The excitation source is placed at $x_{0}=40$ in all cases. The transmission spectra are calculated at $x_{1}=155, x_{1}=251$, and $x_{1}=491$ for $h=6 a, h=10 a$, and $h=20 a$, respectively.

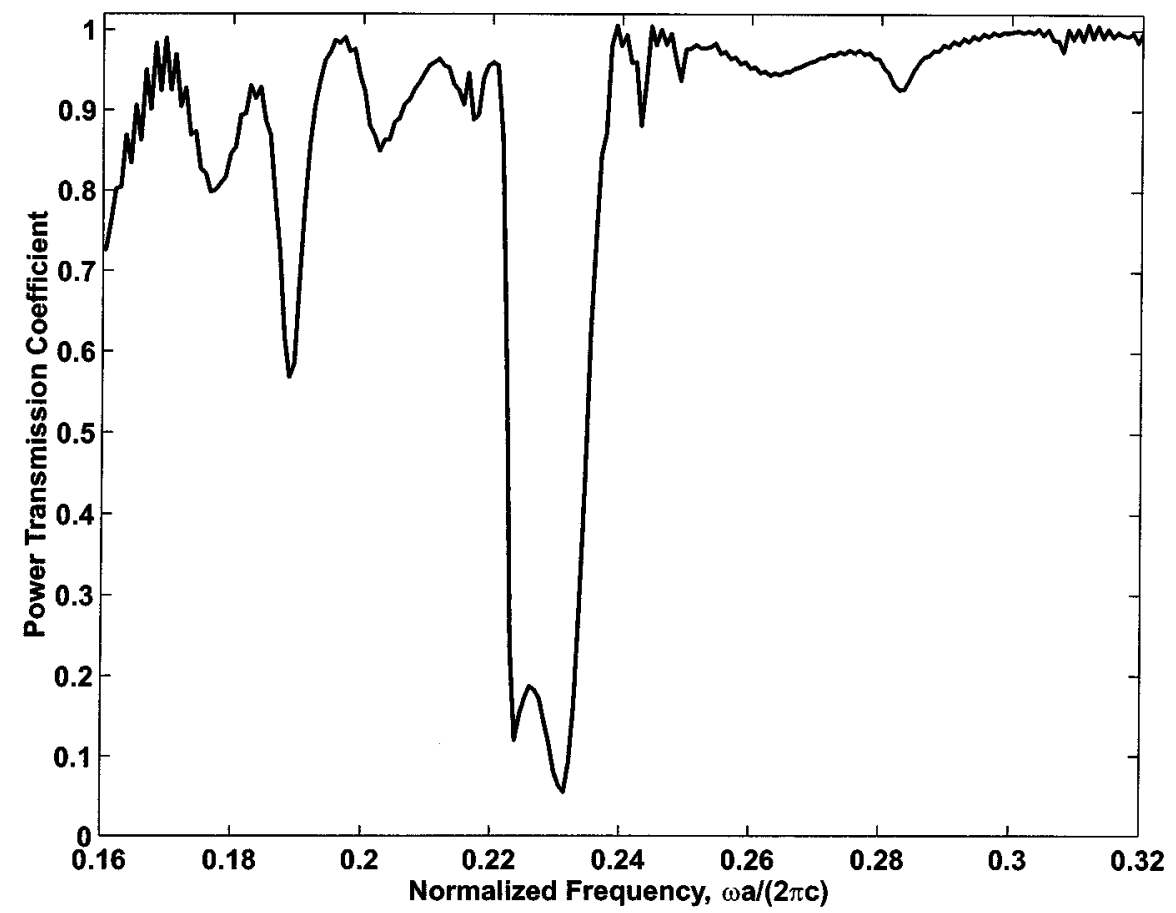

Fig. 12. Transmission spectrum from a slab waveguide to a PBG waveguide with $h=10 a, v=3 a, x_{0}=40$, and $x_{1}=251$. The PBG waveguide is made by removing one row of air holes resulting in $d=27$. The media between the PBG mirrors and the PMLs on the top and bottom of the structure are air in both cases.

\section{CONCLUSION}

We showed here the existence and the properties of the slab modes of PBG waveguides. We showed that these guiding modes can propagate without significant attenuation outside the PBG of the photonic crystal. These modes are similar to the conventional slab waveguide modes, and are practically lossless over a frequency range larger than the PBG. We also showed that we can obtain efficient power transmission from a slab waveguide to a PBG waveguide over a relatively wide frequency range.

The optimization of the PBG waveguide, especially when it is coupled to a slab waveguide can be done by choosing the appropriate slab thickness to guarantee single-mode propagation 
in the frequency range of interest, by shaping the corrugation on both sides of the slab to place the peaks of the DBR outside the desired frequency range, and by designing the photonic crystal on both sides of the slab to have no other (extended) mode in the desired frequency range.

Our results suggest that we can make optical elements using sections of PBG waveguides and use slab waveguides to efficiently connect different PBG optical elements. The results are also significant in explaining the experimental results regarding PBG waveguides. We think that this work defines a new pathway in the design, optimization, and application of the photonic crystal optical waveguides.

\section{REFERENCES}

[1] E. Yablonovitch, "Inhibited spontaneous emission in solid state physics and electronics," Phys. Rev. Lett., vol. 58, pp. 2059-2062, 1987.

[2] S. John, "Strong localization of photons in certain disordered dielectric superlattices," Phys. Rev. Lett., vol. 58, pp. 2486-2489, 1987.

[3] P. Yeh and A. Yariv, "Bragg reflection waveguides," Opt. Commun., vol. 19, pp. 427-430, 1976

[4] A. Mekis, J. C. Chen, I. Kurland, S. Fan, P. R. Villeneuve, and J. D. Joannopoulos, "High transmission through sharp bends in photonic crystal waveguides," Phys. Rev. Lett., vol. 77, pp. 3787-3790, 1996.

[5] S. Lin, E. Chow, V. Hietala, P. R. Villeneuve, and J. D. Joannopoulos, "Experimental demonstration of guiding and bending electromagnetic waves in a photonic crystal," Science, vol. 282, pp. 274-276, 1998.

[6] T. Baba, N. Fukaya, and J. Yonekura, "Observation of light propagation in photonic crystal optical waveguides with bends," Electron. Lett., vol. 35, pp. 654-655, 1999.

[7] M. Tokushima, H. Kosaka, A. Tomita, and H. Yamada, "Lightwave propagation through a 120 degrees sharply bent single-line-defect photonic crystal waveguide," Appl. Phys. Lett., vol. 76, pp. 952-954, 2000.

[8] N. Stefanou and A. Modinos, "Impurity bands in photonic insulators," Phys. Rev. B, vol. 57, pp. 12 127-12133, 1998.

[9] A. Yariv, Y. Xu, R. K. Lee, and A. Scherer, "Coupled-resonator optical waveguide: A proposal and analysis," Opt. Lett., vol. 24, pp. 711-713, 1999.

[10] M. D. B. Charlton, G. J. Parker, and M. E. Zoorob, "Recent developments in the design and fabrication of visible photonic band gap waveguide devices," J. Mater. Sci.-Mater. El., vol. 10, pp. 429-440, 1999.

[11] I. El-Kady, M. M. Sigalas, R. Biswas, and K. M. Ho, "Dielectric waveguides in two-dimensional photonic bandgap materials," J. Lightwave Technol., vol. 17, pp. 2042-2049, 1999.

[12] A. Chutinan and S. Noda, "Highly confined waveguides and waveguide bends in three-dimensional photonic crystal," Appl. Phys. Lett., vol. 75, pp. 3739-3741, 1999.

[13] V. N. Astratov, D. M. Whittaker, I. S. Culshaw, R. M. Stevenson, M. S. Skolnick, T. F. Krauss, and R. M. D. L. Rue, "Photonic band-structure effects in the reflectivity of periodically patterned waveguides," Phys. Rev. $B$, vol. 60, pp. R16255-R16258, 1999.

[14] H. Benisty, D. Labilloy, C. Weisbuch, C. J. M. Smith, T. F. Krauss, D. Cassagne, A. Beraud, and C. Jouanin, "Radiation losses of waveguidebased two-dimensional photonic crystals: Positive role of the substrate," Appl. Phys. Lett., vol. 76, pp. 532-534, 2000.
[15] S. Kuchinsky, D. C. Allan, N. F. Borrelli, and J. C. Cotteverte, "3D localization in a channel waveguide in a photonic crystal with $2 \mathrm{D}$ periodicity," Opt. Commun., vol. 175, pp. 147-152, 2000.

[16] A. Adibi, Y. Xu, R. K. Lee, A. Yariv, and A. Scherer, "Guiding mechanism in dielectic-core crystal optical waveguides," Phys. Rev. B, 2000, submitted for publication.

[17] P. S. J. Russell, "Recent progree in photonic crystal fibers," in $O F C$ $2000,2000$.

[18] J. C. Knight, J. Broeng, T. A. Birks, and P. S. J. Russel, "Photonic band cap guidance in optical fibers," Science, vol. 282, pp. 1476-1478, 1998.

[19] K. S. Yee, "Numerical solution of initial boundary value problems involving Maxwell's equations in isotropic media," IEEE Trans. Antennas Propagat., vol. AP-14, pp. 302-307, 1966.

[20] J. P. Berenger, "A perfectly matched layer for the absorption of electromagnetic waves," J. Comput. Phys., vol. 114, pp. 185-200, 1994.

[21] D. E. Merewether, R. Fisher, and F. W. Smith, "On implementing a numeric Huygen's source scheme in a finite difference program to illuminate scattering bodies," IEEE Trans. Nucl. Sci., vol. NS-27, p. 1829, 1980.

[22] C. T. Chan, Q. L. Yu, and K. M. Ho, "Order-N spectral method for electromagnetic-waves," Phys. Rev. B, vol. 51, pp. 16635-16642, 1995.

[23] Y. Xu, J. S. Vuckovic, R. K. Lee, O. J. Painter, A. Scherer, and A. Yariv, "Finite-difference time-domain calculation of spontaneous emission lifetime in a microcavity," J. Opt. Soc. Amer. B, vol. 16, pp. 465-474, 1999.

[24] Y. Xu, R. K. Lee, and A. Yariv, "Propagation and second-harmonic generation of electromagnetic waves in a coupled-resonator optical waveguide," J. Opt. Soc. Amer. B, vol. 17, pp. 387-400, 2000.

[25] A. Yariv, Optical Electronics in Modern Communications. New York: Oxford Univ. Press, 1996.

[26] J. D. Joannopoulos, R. D. Meade, and J. N. Winn, Photonic Crystals: Molding the Flow of Light. Princeton, NJ: Princeton Univ. Press, 1995.

Ali Adibi, photograph and biography not available at the time of publication.

Yong Xu, photograph and biography not available at the time of publication.

Reginald K. Lee, photograph and biography not available at the time of publication.

Amnon Yariv (LF'95), photograph and biography not available at the time of publication.

Axel Scherer, photograph and biography not available at the time of publication. 